\title{
Ligustrazine reduces blood-brain barrier permeability in a rat model of focal cerebral ischemia and reperfusion
}

\author{
FENG TAN ${ }^{1}$, WENJUN FU ${ }^{2}$, NANFANG CHENG ${ }^{1}$, DI MENG ${ }^{1}$ and YONG GU $^{3}$ \\ ${ }^{1}$ Department of Neurology, Foshan Hospital of Traditional Chinese Medicine, Foshan, Guangdong 528000; \\ ${ }^{2}$ College of Basic Medicine, Guangzhou University of Chinese Medicine, Guangzhou, Guangdong 510405; \\ ${ }^{3}$ Department of Neurology, Nanfang Hospital, Southern Medical University, Guangzhou, Guandong 510515, P.R. China
}

Received June 30, 2014; Accepted March 3, 2015

DOI: $10.3892 / \mathrm{etm} .2015 .2365$

\begin{abstract}
Ligustrazine, also known as 2,3,5,6-tetramethylpyrazine (TMP), one of the major active compounds of Ligusticum wallichii Franchat., has been shown to reduce neuroinflammation and protect neurons during cerebral ischemia/reperfusion injury. However, whether it reduces blood-brain barrier (BBB) permeability during ischemic stroke is unclear. The aim of the present study was to investigate the role that TMP plays in protecting the BBB integrity in ischemia/reperfusion injury and to investigate the relevant mechanisms involved. Rats received an intraperitoneal injection of $20 \mathrm{mg} / \mathrm{kg}$ TMP $15 \mathrm{~min}$ before the onset of ischemia, which was induced by middle cerebral artery occlusion. Infarct volume, neurological score, brain edema, BBB permeability and tight junction protein impairment were observed. The results showed that TMP reduced the neurological score and levels of brain infarction and edema. In addition, TMP significantly decreased BBB permeability and prevented the impairment of occludin and claudin-5, two tight junction protein components of the $\mathrm{BBB}$, in rat brains with ischemia/reperfusion injury. In addition, the expression and activity of matrix metalloproteinases, enzymes responsible for the degradation of the extracellular matrix and tight junctions, were reduced in the rat brains by TMP treatment. These results combined suggest that TMP reduces BBB permeability as well as neuronal damage in focal cerebral ischemia/reperfusion injury in rats.
\end{abstract}

Correspondence to: Dr Feng Tan, Department of Neurology, Foshan Hospital of Traditional Chinese Medicine, 6 Qinren Road, Foshan, Guangdong 528000, P.R. China

E-mail: tanfeng8208@163.com

Dr Yong Gu, Department of Neurology, Nanfang Hospital, Southern Medical University, 1838 Guangzhou Avenue North, Guangzhou, Guangdong 510515, P.R. China

E-mail: yonggu@smu.edu.cn

Key words: ligustrazine, tetramethylpyrazine, blood-brain barrier, cerebral ischemia, tight junction

\section{Introduction}

Current therapeutic approaches for ischemic stroke mainly focus on the re-canalization of occluded blood vessels in the brain; however, reperfusion following thrombolytic therapy induces a cascade of pathological events, including an inflammatory response and blood-brain barrier (BBB) breakdown $(1,2)$. BBB injury is the key event that leads to vascular edema and hemorrhage transformation, two high-risk factors for intracranial hypertension and brain death $(1,3,4)$. Thus, preventing BBB breakdown is a promising strategy to halt the progression of ischemic stroke.

In Traditional Chinese Medicine, Ligusticum wallichii Franchat. (Chuan Xiong) is widely used in the treatment of cardiovascular and neurovascular diseases. Ligustrazine (Fig. 1), also known as 2,3,5,6-tetramethylpyrozine (TMP), is one of the most important active ingredients of Chuan Xiong (5). The compound can penetrate BBB and can be found throughout the brain. Chuan Xiong and TMP are widely applied by Chinese herbalists to treat ischemic stroke (6). Several animal studies have demonstrated that TMP is capable of reducing the infarct volume, neurological score and brain edema in the model of permanent and temporal cerebral ischemia injuries (7-11). TMP also reduces cellular inflammatory responses that follow brain ischemia (11) and enhances neurogenesis and spatial cognition during ischemic injury (8). The neuroprotective effect of TMP has been confirmed by multiple in vitro studies $(12,13)$. TMP has been shown to have neuroprotective effects against Parkinson's disease (14), neuropathic pain (15) and spinal cord ischemia (16).

With regard to the mechanism underlying the TMP-induced neuroprotection, TMP has been reported to possess diverse pharmacological properties, including free radical scavenging and the inhibition of cytokine production and release (17-19). The inflammatory response and free radical production during cerebral ischemia and reperfusion are the main causes of BBB breakdown. Thus, it is possible that TMP could protect the $\mathrm{BBB}$ during cerebral ischemia and reperfusion, due to its association with free radical scavenging and inflammatory inhibition. In the present study, a classical brain ischemia and reperfusion model was utilized to investigate the neuroprotective and BBB-protective role of TMP. The relevant mechanisms involved in the effects of TMP were additionally investigated. 


\section{Materials and methods}

Ethics statement. Male adult Sprague Dawley rats (250-270 g) were obtained from the Animal Center of the Guangzhou University of Chinese Medicine (Guangzhou, China). The present study was conducted in strict accordance with the international ethical guidelines and the Guide for the Care and Use of Laboratory Animals by the National Institutes of Health. All animal experiments were approved by the Institutional Animal Care and Use Committee of Guangzhou University of Chinese Medicine. Every effort was made to minimize the number and suffering of the animals used.

Middle cerebral artery occlusion (MCAO) and drug treatment. MCAO was selected as the model for cerebral ischemia/reperfusion (20). Briefly, anesthesia was induced in the rats through the inhalation of 5\% isoflurane and maintained with $2 \%$ isoflurane in a mixture of $70 \% \mathrm{~N}_{2} \mathrm{O}$ and $30 \% \mathrm{O}_{2}$. Following vessel isolation, a 3-0 monofilament nylon suture (Johnson \& Johnson, New Brunswick, NJ, USA) was inserted into the external carotid artery and then threaded forward into the internal carotid artery and anterior cerebral artery to occlude the MCA. Following the surgery, the rats were transferred to an intensive care incubator where the temperature was maintained at $37^{\circ} \mathrm{C}$ until the animals regained consciousness. The suture was removed following a 1.5 -h occlusion period, in order to induce reperfusion. TMP $(20 \mathrm{mg} / \mathrm{kg}$; Forever Biotech, Shanghai, China) (Fig. 1) or a saline vehicle was intraperitoneally injected into the rats $15 \mathrm{~min}$ before suture insertion. Following $22.5 \mathrm{~h}$ of reperfusion, each rat brain was isolated and subjected to infarct volume, BBB permeability and brain edema measurement, as well as protein preparation for western blotting and the determination of matrix metalloproteinase (MMP) activity (Fig. 2).

Measurement of infarct volume, water content and neurological score. Following reperfusion, each rat brain was isolated and sliced into 5 coronal sections $(2-\mathrm{mm})$, which were stained with 2,3,5-triphenyltetrazolium chloride (Sigma, St. Louis, MO, USA). The evaluation of the infarct area was accomplished by calculating the hemispheric lesion area with ImageJ-1.38x software (National Institutes of Health, Bethesda, MD, USA). The relative infarct volume percentage (RIVP) was calculated using the following formula: RIVP = IVA - TA x 100\% (IVA, total infracted area of the 5 coronal sections; TA, total area of the 5 sections).

Neurological evaluation was performed at $3 \mathrm{~h}$ after occlusion and scored as follows: 0 , no neurological deficit; 1 , difficulty in fully extending the left forepaw; 2 , unable to extend the left forepaw; 3 , mild circling to the left; 4 , severe circling to the left; and 5, falling to the left.

In order for the brain water content to be determined, the isolated brain samples were dried in an oven at $110^{\circ} \mathrm{C}$ for $24 \mathrm{~h}$, and the water content of these samples was subsequently evaluated using the following formula: Water content $(\%)=$ [wet weight - dry weight] - wet weight x 100.

$B B B$ permeability evaluation. BBB permeability was evaluated by measuring the Evans blue dye extravasation in accordance with a previously described method with certain

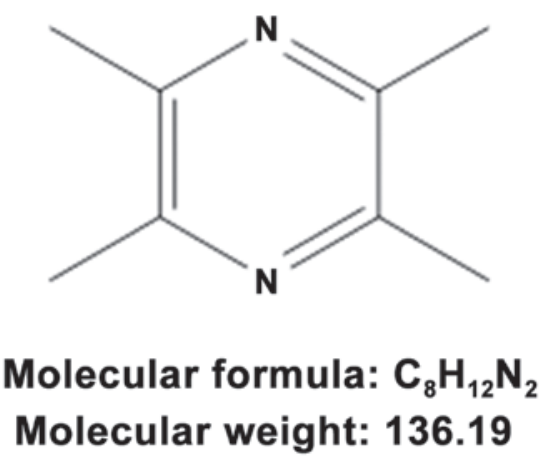

Figure 1. Chemical structure of ligustrazine (2,3,5,6-tetramethylpyrazine).

modifications (21). Briefly, 2\% Evans blue was injected intravenously ( $2 \mathrm{ml} / \mathrm{kg}$, Sigma) $0.5 \mathrm{~h}$ before sacrifice; transcardial saline perfusion was then performed for the removal of all intravascular dye from the vessels, until the drainage was colorless. The ipsilateral hemisphere was then removed and incubated for $24 \mathrm{~h}$ in N,N'-dimethyl formamide (Sigma) in a water bath at $60^{\circ} \mathrm{C}$, and a spectrophometer (Analytik Jena AG, Jena, Germany) was used to measure the Evans blue content in the supernatants at a wavelength of $632 \mathrm{~nm}$. Rats were euthanized after $22.5 \mathrm{~h}$ of reperfusion, by an intraperitoneal injection of pentobarbitone $(200 \mathrm{mg} / \mathrm{ml})$.

In situ zymography. The kit used for the analysis of the gelatinolytic activities of MMP-2 and -9 in cryosections from brain tissues by in situ zymography was the EnzChek ${ }^{\circledR}$ collagenase kit (Invitrogen Life Technologies, Carlsbad, CA, USA); the manufacturer's instructions were followed. Brain coronal cryosections were incubated in a reaction buffer, which contained $40 \mu \mathrm{g} / \mathrm{ml}$ fluorescein isothiocyanate-labeled dye-quenched (DQ)-gelatin, at $37^{\circ} \mathrm{C}$ for $2 \mathrm{~h}$. Gelatinases cleaved the DQ-gelatin to yield the peptides and form fluorescence, which was representative of the net gelatinolytic activity. A fluorescence microscope (Carl Zeiss AG, Oberkochen, Germany) was used to examine the fluorescent imaging.

Western blot analysis. Denatured protein samples were resolved using SDS-PAGE and transferred to polyvinylidene difluoride membrane (Millipore, Billerica, MA, USA). Subsequent to blocking, the membrane was incubated overnight at $4^{\circ} \mathrm{C}$, with rabbit polyclonal anti-MMP-9 (\#3852; 1:500; Cell Signaling Technology, Inc., Boston, MA, USA) and anti-occludin (\#71-1500; 1:1,000; Invitrogen Life Technologies), and mouse monoclonal anti-claudin-5 (\#4C3C2; 1:500; Invitrogen Life Technologies) and anti- $\beta$-actin (\#sc-47778; $1: 2,000$; Santa Cruz Biotechnology Inc., Santa Cruz, CA, USA) primary antibodies. The membrane was then incubated with goat anti-mouse or anti-rabbit horseradish peroxidase-conjugated secondary antibodies (1:2,000; Santa Cruz Biotechnology Inc.). ECL Advance ${ }^{\mathrm{TM}}$ western blotting detection reagents (GE Healthcare, Little Chalfont, UK) were used to perform immunodetection.

Statistical analysis. Data are expressed as the mean \pm standard deviation. For two-group experiments, comparisons were made using an unpaired Student's t-test. SPSS 16.0 statistical 


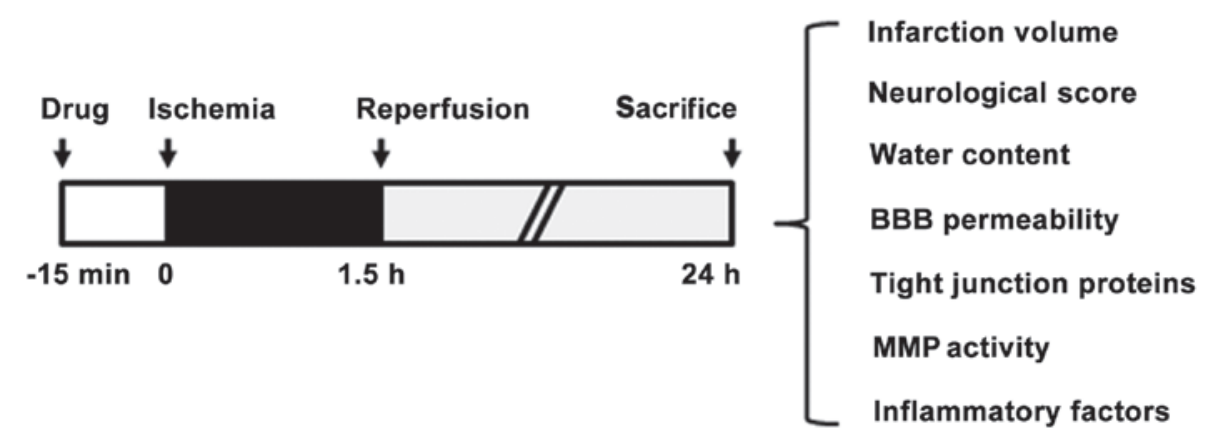

Figure 2. Outline of the experimental design showing the time scale of drug administration and cerebral ischemia and reperfusion. Rats were administered $20 \mathrm{mg} / \mathrm{kg} \mathrm{2,3,5,6-tetramethylpyrazine} \mathrm{(or} \mathrm{saline} \mathrm{vehicle)} 15 \mathrm{~min}$ before the induction of ischemia. After $1.5 \mathrm{~h}$ of ischemia plus $22.5 \mathrm{~h}$ of reperfusion, the rat brains were isolated and subjected to infarct volume, brain edema, MMP activity and tight junction impairment evaluations. BBB, blood-brain barrier; MMP, matrix metalloproteinase.

A
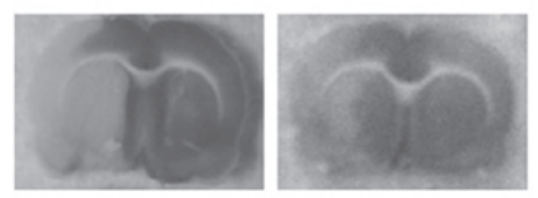

C

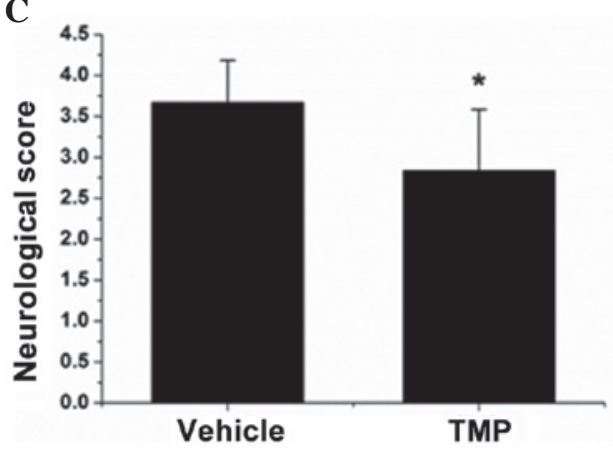

B

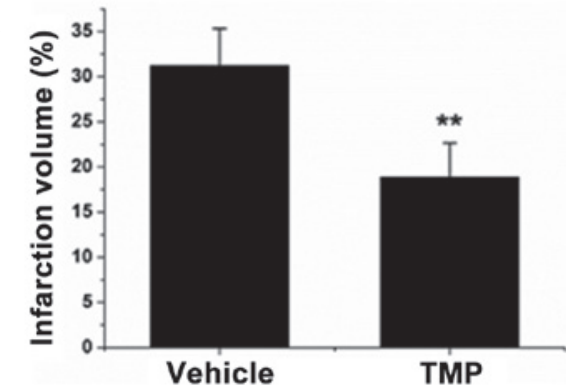

D

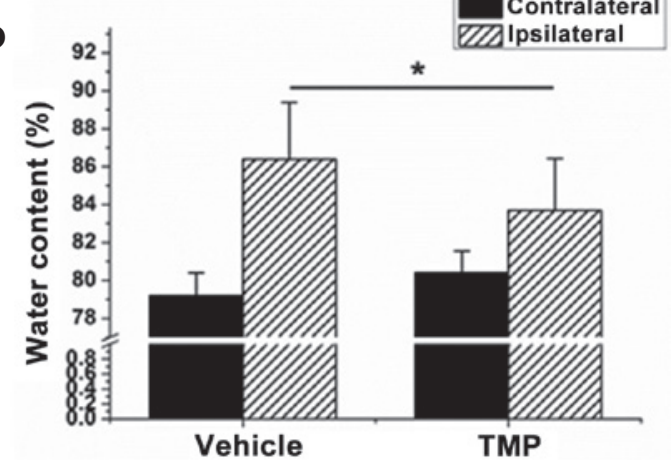

Figure 3. TMP reduces the infarct volume, neurological score and brain edema during cerebral ischemia and reperfusion in rats. (A) The infarct volume of each treatment group was assayed by 2,3,5-triphenyltetrazolium chloride staining. (B) The relative infarct volume percentage was calculated with ImageJ-1.38x software (National Institutes of Health, Bethesda, MD, USA) and presented as a bar graph. (C) The neurological score of each rat was determined and recorded. (D) The water content was calculated according to the difference between the wet and dry weight, and presented as a bar graph. Data are expressed as the mean \pm standard deviation, $n=8 .{ }^{* *} \mathrm{P}<0.01,{ }^{*} \mathrm{P}<0.05$. TMP, 2,3,5,6-tetramethylpyrazine.

software (SPSS, Inc., Chicago, IL, USA) was used for the statistical analysis. $\mathrm{P}<0.05$ was considered to indicate a statistically significant difference.

\section{Results}

Reduced infarct volume, neurological score and brain edema in a rat model of cerebral ischemia/reperfusion injury following TMP treatment. The first experiment was performed to investigate the neuroprotective effect of TMP during cerebral ischemia and reperfusion. As shown in Fig. 3A and B, the brains subjected to ischemia/reperfusion injury exhibited a maximal infarct volume of $31.2 \pm 4.14 \%$, whereas following treatment with TMP that volume dropped to $18.9 \pm 3.75 \%$ $(\mathrm{P}<0.01)$. TMP was also found to reduce the neurological score and brain edema in brains with ischemia/reperfusion injury $(\mathrm{P}<0.05$, Fig. $3 \mathrm{C}$ and $\mathrm{D})$. These results reveal that TMP plays a neuroprotective role against cerebral ischemia/reperfusion injury.

Decreased BBB permeability during rat cerebral ischemia and reperfusion following TMP treatment. BBB leakage is the characteristic feature of cerebral ischemia/reperfusion injury, contributing to the formation of brain edema and hemorrhage. Based on the effect of the TMP-induced reduction in brain edema in rats with cerebral ischemia/reperfusion injuries, the next experiment was performed to examine the effect of TMP on BBB integrity. The results demonstrated that TMP significantly reduced the BBB permeability (from $9.49 \pm 1.97$ to $4.82 \pm 1.14 \mu \mathrm{g} / \mathrm{g}$ brain) $(\mathrm{P}<0.01$, Fig. $4 \mathrm{~A})$. The impairment 
A

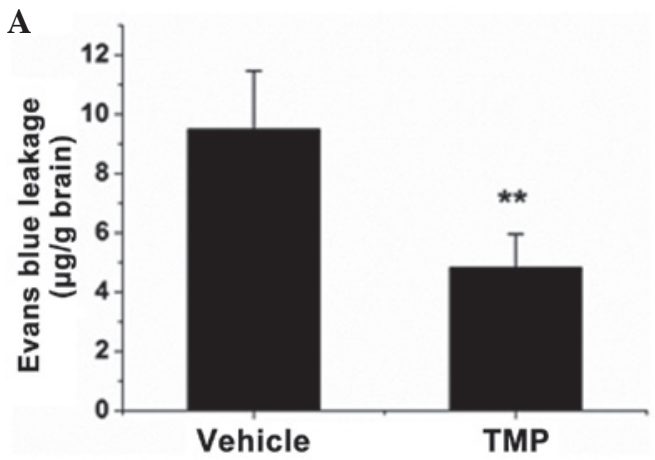

B

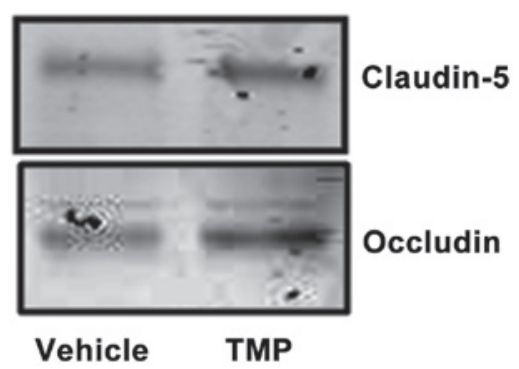

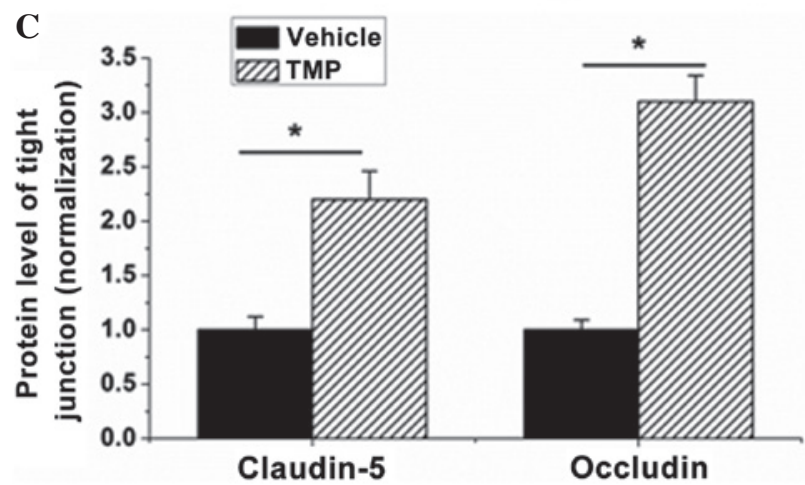

Figure 4. TMP decreases BBB permeability and tight junction impairment in brains with cerebral ischemia/reperfusion injury. (A) BBB permeability in each group was evaluated by measuring the Evans blue extravasation. (B) Western blot analysis was used to determine the expression of occludin and claudin-5 in the protein samples of the brains isolated from the TMP- or saline-treated rats. (C) The blots of occludin and claudin-5 were quantified with ImageJ-1.38x software (National Institutes of Health, Bethesda, MD, USA) and presented in the bar graph. Data are expressed as the mean \pm standard deviation, $\mathrm{n}=6$. ${ }^{* *} \mathrm{P}<0.01,{ }^{*} \mathrm{P}<0.05$. TMP, 2,3,5,6-tetramethylpyrazine; BBB, blood-brain barrier.

A

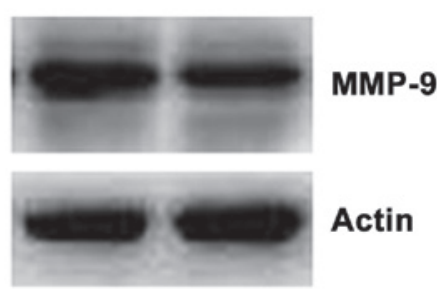

B

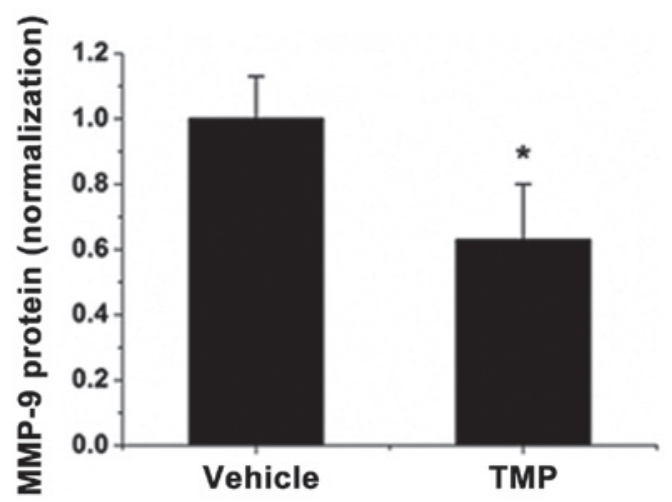

C

MMP activity

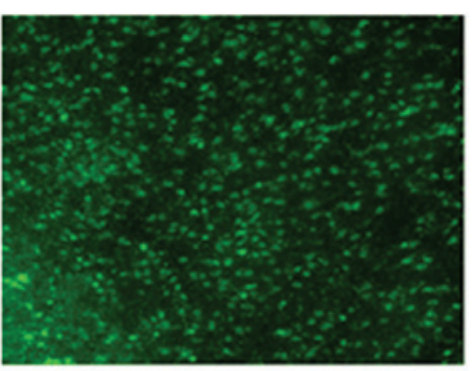

Vehicle

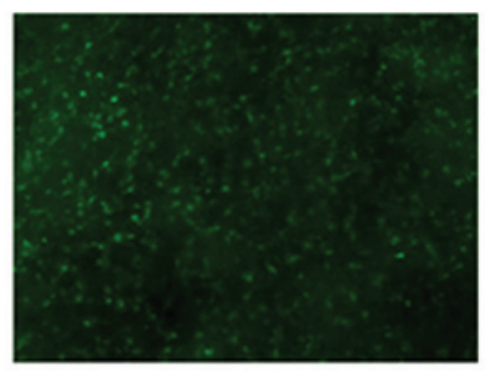

TMP

Figure 5. TMP reduces MMP-9 expression and MMP activity in the brain. (A) The MMP-9 expression level in the brains of TMP- or saline-treated rats was determined by western blotting. (B) Quantitative data of the western blot bands of MMP-9 expression. The average band intensity of MMP-9 was quantified with ImageJ-1.38x software (National Institutes of Health, Bethesda, MD, USA) and presented as a bar graph. (C) MMP activity in cryosections in each group was determined in situ, using a fluorescence-conjugated substrate. Data are expressed as the mean \pm standard deviation, $\mathrm{n}=6$. ${ }^{*} \mathrm{P}<0.05$. TMP, 2,3,5,6-tetramethylpyrazine; MMP, matrix metalloproteinase. 
of tight junctions plays the key role in BBB opening, and it was observed that the expression of occludin and claudin-5, two tight junction proteins, was significantly elevated in the ischemia/reperfusion-injured brains in the TMP treatment group compared with that in the vehicle group $(\mathrm{P}<0.05$, Fig. 4B and C). These data indicate that TMP can significantly reduce $\mathrm{BBB}$ permeability during cerebral ischemia/reperfusion injury.

Decreased MMP activity during rat cerebral ischemia and reperfusion following TMP treatment. MMPs are a group of zinc-dependent proteinases responsible for the matrix and tight junction degradation (22). MMP activation and the secondary impairment of tight junction proteins are the main causes of BBB leakage. In order to study the relevant mechanisms involved in BBB protection, the MMP activities in brain sections and the MMP-9 expression were observed. Fig. 5A and B show that TMP treatment reduced the protein level of MMP-9. Correspondingly, TMP markedly reduced the elevated MMP activity in the brains of rats subjected to MCAO, as shown by in situ zymography with a fluorescence-conjugated substrate (Fig. 5C). These results suggest that TMP decreased the MMP activity during rat cerebral ischemia and reperfusion.

\section{Discussion}

The present study investigated the effects of TMP at a neurovascular level and found that i) TMP simultaneously reduced the infarct volume, neurological score, brain edema and $\mathrm{BBB}$ breakdown in a rat model of cerebral ischemia and reperfusion, a finding that is consistent with previously reported findings; ii) TMP reduced BBB permeability in the brain of rats with ischemia/reperfusion damage; iii) activated MMPs and impaired tight junctions in ischemic brains were significantly attenuated by TMP treatment.

During cerebral ischemia and reperfusion, toxic substances can readily pass from the blood to the brain due to the increased permeability of the $\mathrm{BBB}$ induced by reperfusion insults; this results in an enlarged infarct volume. Thus, maintaining BBB integrity is a critical therapeutic strategy for the prevention of cerebral ischemia/reperfusion injury. The most notable finding of the present study is that TMP can protect the BBB integrity during cerebral ischemia and reperfusion.

The BBB is composed of extracellular matrix, microvascular endothelial cells and astrocytic endfeet. The primary responsibility of the $\mathrm{BBB}$ is the strict regulation of trans-BBB permeability. In this regard, the endothelial tight junctions of the capillary are the main mediators, restricting the transfer of vascular-derived substances (23). The most important membrane-associated tight junction proteins include occludins, claudins and junctional adhesion molecules, which are connected to the cytoskeleton by the zonula occludens $(24,25)$. Elevated MMP activity and tight junctional impairment are typical events associated with the BBB dysfunction during cerebral ischemia/reperfusion. The in situ zymography assay and the western blot analysis found that TMP treatment reduced MMP activity, decreased MMP-9 expression and attenuated the loss of the tight junction proteins occludin and claudin-5. These results further confirmed that TMP was capable of protecting the $\mathrm{BBB}$ from increased permeability during cerebral ischemia and reperfusion.

In conclusion, this study is the first, to the best of our knowledge, to demonstrate that TMP can preserve the BBB integrity in this model of ischemic stroke in rats. The mechanisms underlying this protection could be associated with the regulation of MMPs and tight junctions. The findings of the present study could be applied in a therapeutic or preventive strategy for patients that have or are at high risk of suffering a stroke.

\section{Acknowledgements}

This study was supported by the National Natural Science of Foundation of China (no. 81072947), the Guangdong Natural Science Foundation (no. 8152800007000001) and the Collaborative Innovation Center for Key Technology of the Wudang Genuine Medicines Industry in Hubei Province (no. 2011JH-2103CXTT08).

\section{References}

1. Gu Y, Dee CM and Shen J: Interaction of free radicals, matrix metalloproteinases and caveolin-1 impacts blood-brain barrier permeability. Front Biosci (Schol Ed) 3: 1216-1231, 2011.

2. Eltzschig $\mathrm{HK}$ and Eckle T: Ischemia and reperfusion - from mechanism to translation. Nat Med 17: 1391-1401, 2011.

3. Gu Y, Chen J and Shen J: Herbal medicines for ischemic stroke: Combating inflammation as therapeutic targets. J Neuroimmune Pharmacol 9: 313-339, 2014.

4. Obermeier B, Daneman R and Ransohoff RM: Development, maintenance and disruption of the blood-brain barrier. Nat Med 19: 1584-1596, 2013.

5. Han JZ, Sun J, Zhu QG, Liu JY, Hu JH and Chen F: A modified LC-MS/MS method for determination of tetramethylpyrazine in microdialysis samples and calibration of home-made linear probes. Biomed Chromatogr 26: 1276-1281, 2012.

6. Chun-sheng L, Hsiao-meng Y, Yun-hsiang H, Chun P and Chi-fen S: Radix Salviae Miltiorrhizae and Rhizoma Ligustici Wallichii in coronary heart disease. Chin Med J (Engl) 4: 43-46, 1978.

7. Zhu XL, Xiong LZ, Wang Q,Liu ZG, Ma X, Zhu ZH, Hu S, Gong G and Chen SY: Therapeutic time window and mechanism of tetramethylpyrazine on transient focal cerebral ischemia/reperfusion injury in rats. Neurosci Lett 449: 24-27, 2009.

8. Kao TK, Ou YC, Kuo JS, Chen WY, Liao SL, Wu CW, Chen CJ, Ling NN, Zhang YH and Peng WH: Neuroprotection by tetramethylpyrazine against ischemic brain injury in rats. Neurochem Int 48: 166-176, 2006

9. Xiao X, Liu Y, Qi C, Qiu F, Chen X, Zhang J and Yang P: Neuroprotection and enhanced neurogenesis by tetramethylpyrazine in adult rat brain after focal ischemia. Neurol Res 32: 547-555, 2010.

10. Liao SL, Kao TK, Chen WY, Lin YS, Chen SY, Raung SL, $\mathrm{Wu} \mathrm{CW}, \mathrm{Lu} \mathrm{HC}$ and Chen CJ: Tetramethylpyrazine reduces ischemic brain injury in rats. Neurosci Lett 372: 40-45, 2004.

11. Kao TK, Chang CY, Ou YC, Chen WY, Kuan YH, Pan HC, Liao SL, Li GZ and Chen CJ: Tetramethylpyrazine reduces cellular inflammatory response following permanent focal cerebral ischemia in rats. Exp Neurol 247: 188-201, 2013.

12. Shih YH, Wu SL, Chiou WF, Ku HH, Ko TL and Fu YS: Protective effects of tetramethylpyrazine on kainate-induced excitotoxicity in hippocampal culture. Neuroreport 13: 515-519, 2002.

13. Li SY, Jia YH, Sun WG, Tang Y, An GS, Ni JH and Jia HT: Stabilization of mitochondrial function by tetramethylpyrazine protects against kainate-induced oxidative lesions in the rat hippocampus. Free Radic Biol Med 48: 597-608, 2010.

14. Lu C, Zhang J, Shi X, Miao S, Bi L, Zhang S, Yang Q, Zhou X, Zhang M, Xie Y, et al: Neuroprotective effects of tetramethylpyrazine against dopaminergic neuron injury in a rat model of Parkinson's disease induced by MPTP. Int J Biol Sci 10: 350-357, 2014. 
15. Leng YF, Gao XM, Wang SX and Xing YH: Effects of tetramethylpyrazine on neuronal apoptosis in the superficial dorsal horn in a rat model of neuropathic pain. Am J Chin Med 40: 1229-1239, 2012.

16. Fan LH, Wang KZ, Cheng B, Wang CS and Dang XQ: Anti-apoptoticand neuroprotective effects of Tetramethylpyrazine following spinal cord ischemia in rabbits. BMC Neurosci 7: 48, 2006.

17. Feng L, Ke N, Cheng F, Guo Y, Li S, Li Q and Li Y: The protective mechanism of ligustrazine against renal ischemia/reperfusion injury. J Surg Res 166: 298-305, 2011.

18. Chang Y, Hsiao G, Chen SH, Chen YC, Lin JH, Lin KH, Chou DS and Sheu JR: Tetramethylpyrazine suppresses HIF-1alpha, TNF-alpha, and activated caspase-3 expression in middle cerebral artery occlusion-induced brain ischemia in rats. Acta Pharmacol Sin 28: 327-333, 2007.

19. Li XY, He JL, Liu HT, Li WM and Yu C: Tetramethylpyrazine suppresses interleukin-8 expression in LPS-stimulated human umbilical vein endothelial cell by blocking ERK, p38 and nulear factor-kappaB signaling pathways. J Ethnopharmacol 125: 83-89, 2009.
20. Gu Y, Zheng G, Xu M, et al: Caveolin-1 regulates nitric oxide-mediated matrix metalloproteinases activity and blood-brain barrier permeability in focal cerebral ischemia and reperfusion injury. J Neurochem 120: 147-156, 2012.

21. Yu F, Kamada H, Niizuma K, Endo H and Chan PH: Induction of mmp-9 expression and endothelial injury by oxidative stress after spinal cord injury. J Neurotrauma 25: 184-195, 2008.

22. Candelario-Jalil E, Thompson J, Taheri S, Grossetete M, Adair JC, Edmonds E, Prestopnik J, Wills J and Rosenberg GA: Matrix metalloproteinases are associated with increased blood-brain barrier opening in vascular cognitive impairment. Stroke 42: 1345-1350, 2011.

23. Vorbrodt AW and Dobrogowska DH: Molecular anatomy of intercellular junctions in brain endothelial and epithelial barriers: Electron microscopist's view. Brain Res Brain Res Rev 42: 221-242, 2003.

24. Sandoval KE and Witt KA: Blood-brain barrier tight junction permeability and ischemic stroke. Neurobiol Dis 32: 200-219, 2008.

25. Krizbai IA and Deli MA: Signalling pathways regulating the tight junction permeability in the blood-brain barrier. Cell Mol Biol (Noisy-le-grand) 49: 23-31, 2003. 